\title{
Planned Number of Arms
}

National Cancer Institute

\section{Source}

National Cancer Institute. Planned Number of Arms. NCI Thesaurus. Code C98771.

The planned number of intervention groups. 